\title{
CHARACTERIZATIONS OF RAPIDLY DECREASING GENERALIZED FUNCTIONS
}

\author{
Chikh Bouzar and Tayeb Saidi
}

\begin{abstract}
The well-known characterizations of the Schwartz space of rapidly decreasing functions is extended to new algebras of rapidly decreasing generalized functions.
\end{abstract}

\section{Introduction}

The Schwartz space $\mathcal{S}$ of rapidly decreasing functions on $\mathbb{R}^{n}$ and its different generalizations, in view of their importance in analysis, have been characterized differently by many authors, e.g. see [17], [12], [14], [4], [9] and [1]. Recall that

and let

$$
\mathcal{S}=\left\{f \in \mathcal{C}^{\infty}: \forall \alpha \in \mathbb{Z}_{+}^{n}, \forall \beta \in \mathbb{Z}_{+}^{n}, \sup _{x \in \mathbb{R}^{n}}\left|x^{\beta} \partial^{\alpha} f(x)\right|<\infty\right\}
$$

$$
\begin{aligned}
& \mathcal{S}^{*}=\left\{f \in \mathcal{C}^{\infty}: \forall \alpha \in \mathbb{Z}_{+}^{n}, \sup _{x \in \mathbb{R}^{n}}\left|\partial^{\alpha} f(x)\right|<\infty\right\}, \\
& \mathcal{S}_{*}=\left\{f \in \mathcal{C}^{\infty}: \forall \beta \in \mathbb{Z}_{+}^{n}, \sup _{x \in \mathbb{R}^{n}}\left|x^{\beta} f(x)\right|<\infty\right\},
\end{aligned}
$$

then the characterization of $\mathcal{S}$ given in [4] is the result:

$$
\mathcal{S}=\mathcal{S}^{*} \cap \mathcal{S}_{*} .
$$

To built a Fourier analysis within the new generalized functions of [5], the algebra of rapidly decreasing generalized functions on $\mathbb{R}^{n}$, denoted $\mathcal{G}_{\mathcal{S}}$, was first constructed in [15] and recently studied in [7] and [6]. The algebra of regular rapidly decreasing generalized functions on $\mathbb{R}^{n}$, denoted $\mathcal{G}_{\mathcal{S}}^{\infty}$, is fundamental in the study of local regularity of a Colombeau generalized functions and also for developing a generalized microlocal analysis.

The purpose of this work is to lift the characterizations of the Schwartz space $\mathcal{S}=\mathcal{S}^{*} \cap \mathcal{S}_{*}$ to the algebras $\mathcal{G}_{\mathcal{S}}$ and $\mathcal{G}_{\mathcal{S}}^{\infty}$. Actually we do more, these characterizations are given in the general context of the algebras $\mathcal{G}_{\mathcal{S}}^{\mathcal{R}}(\Omega)$ of

Received May 6, 2009; Revised April 22, 2010.

2000 Mathematics Subject Classification. 46F30, 46F05, 42B10.

Key words and phrases. Schwartz space, rapidly decreasing generalized functions, Colombeau algebra, Fourier transform. 
$\mathcal{R}$-rapidly decreasing generalized functions on an open set $\Omega$ of $\mathbb{R}^{n}$, see $[6]$ and [2]. Section 2 recall the notion of regular set of sequences. Sections 3,4 and 5 introduce, respectively, the algebra of $\mathcal{R}$-bounded generalized functions, the algebra of $\mathcal{R}$-roughly decreasing generalized functions and the algebra of $\widetilde{\mathcal{R}}$ rapidly decreasing generalized functions. Section 6 gives the characterization of the algebra $\mathcal{G}_{\mathcal{S}}^{\widetilde{\mathcal{R}}}(\Omega)$, provided $\Omega$ is a box of $\mathbb{R}^{n}$, and as corollaries of this result we obtain the characterizations of the classical algebras $\mathcal{G}_{\mathcal{S}}$ and $\mathcal{G}_{\mathcal{S}}^{\infty}$. Finally, Section 7 gives the characterization of $\mathcal{G}_{\mathcal{S}}^{\widetilde{\mathcal{R}}}\left(\mathbb{R}^{n}\right)$ using the Fourier transform.

\section{Regular set of sequences}

Recall the definition of a regular set of sequences introduced in [6], see [2].

Definition. A non void subset $\mathcal{R}$ of $\mathbb{R}_{+}^{\mathbb{Z}_{+}}$is said to be regular, if that

For all $\left(N_{m}\right)_{m \in \mathbb{Z}_{+}} \in \mathcal{R}$ and $\left(k, k^{\prime}\right) \in \mathbb{Z}_{+}^{2}$, there exists $\left(N_{m}^{\prime}\right)_{m \in \mathbb{Z}_{+}} \in \mathcal{R}$ such

$$
N_{m+k}+k^{\prime} \leq N_{m}^{\prime}, \forall m \in \mathbb{Z}_{+} .
$$
that

For all $\left(N_{m}\right)_{m \in \mathbb{Z}_{+}}$and $\left(N_{m}^{\prime}\right)_{m \in \mathbb{Z}_{+}}$in $\mathcal{R}$, there exists $\left(N{ }^{\prime}\right)_{m \in \mathbb{Z}_{+}} \in \mathcal{R}$ such

$$
\max \left(N_{m}, N_{m}^{\prime}\right) \leq N^{\prime \prime}{ }_{m}, \forall m \in \mathbb{Z}_{+} .
$$

For all $\left(N_{m}\right)_{m \in \mathbb{Z}_{+}}$and $\left(N_{m}^{\prime}\right)_{m \in \mathbb{Z}_{+}}$in $\mathcal{R}$, there exists $\left(N^{\prime \prime}\right)_{m \in \mathbb{Z}_{+}} \in \mathcal{R}$ such that

$$
N_{m_{1}}+N_{m_{2}}^{\prime} \leq N^{\prime \prime}{ }_{m_{1}+m_{2}}, \forall\left(m_{1}, m_{2}\right) \in \mathbb{Z}_{+}^{2} .
$$

The notion of regular set is extended to the sets of double sequences.

Definition. A non void subset $\widetilde{\mathcal{R}}$ of $\mathbb{R}_{+}^{\mathbb{Z}_{+}^{2}}$ is said to be regular if

For all $\left(N_{q, l}\right)_{(q, l) \in \mathbb{Z}_{+}^{2}} \in \widetilde{\mathcal{R}}$ and $\left(k, k^{\prime}, k^{\prime \prime}\right) \in \mathbb{Z}_{+}^{3}$, there exists $\left(N_{q, l}^{\prime}\right)_{(q, l) \in \mathbb{Z}_{+}^{2}} \in$ $\widetilde{\mathcal{R}}$ such that

$$
N_{q+k, l+k^{\prime}}+k^{\prime \prime} \leq N_{q, l}^{\prime}, \forall(q, l) \in \mathbb{Z}_{+}^{2}
$$

For all $\left(N_{q, l}\right)_{(q, l) \in \mathbb{Z}_{+}^{2}}$ and $\left(N_{q, l}^{\prime}\right)_{(q, l) \in \mathbb{Z}_{+}^{2}}$ in $\widetilde{\mathcal{R}}$, there exists $\left(N^{\prime \prime}{ }_{q, l}\right)_{(q, l) \in \mathbb{Z}_{+}^{2}} \in$ $\widetilde{\mathcal{R}}$ such that

$$
\max \left(N_{q, l}, N_{q, l}^{\prime}\right) \leq N^{\prime \prime}{ }_{q, l}, \forall(q, l) \in \mathbb{Z}_{+}^{2}
$$

For all $\left(N_{q, l}\right)_{(q, l) \in \mathbb{Z}_{+}^{2}}$ and $\left(N_{q, l}^{\prime}\right)_{(q, l) \in \mathbb{Z}_{+}^{2}}$ in $\widetilde{\mathcal{R}}$, there exists $\left(N^{\prime \prime}{ }_{q, l}\right)_{(q, l) \in \mathbb{Z}_{+}^{2}} \in$ $\widetilde{\mathcal{R}}$ such that

$$
N_{q_{1}, l_{1}}+N_{q_{2}, l_{2}}^{\prime} \leq N^{\prime \prime}{ }_{q_{1}+q_{2}, l_{1}+l_{2}}, \forall\left(q_{1}, q_{2}, l_{1}, l_{2}\right) \in \mathbb{Z}_{+}^{4} .
$$


Example 2.1. i) The set $\mathbb{R}_{+}^{\mathbb{Z}_{+}}$of all positive sequences is regular.

ii) The set $\mathcal{A}$ of affine sequences defined by

$$
\mathcal{A}=\left\{\left(N_{m}\right)_{m \in \mathbb{N}} \in \mathbb{R}_{+}^{\mathbb{Z}_{+}}: \exists a \geq 0, \exists b \geq 0, \forall m \in \mathbb{Z}_{+}, N_{m} \leq a m+b\right\}
$$

is regular.

iii) The set $\mathcal{B}$ of all bounded sequences of $\mathbb{R}_{+}^{\mathbb{Z}_{+}}$is regular.

iv) The set $\mathbb{R}_{+}^{\mathbb{Z}_{+}^{2}}$ of all positive double sequences is regular.

v) The set $\widetilde{\mathcal{B}}$ of all bounded sequences of $\mathbb{R}_{+}^{\mathbb{Z}_{+}^{2}}$ is regular.

We give the following results, easy to prove, needed in the formulation of the principal theorems of this paper.

Lemma 2.2. Let $\widetilde{\mathcal{R}}$ be a regular subset of $\mathbb{R}_{+}^{\mathbb{Z}_{+}^{2}}$. Then

(i) The subset $\widetilde{\mathcal{R}}^{0}:=\left\{N_{., 0}: N \in \widetilde{\mathcal{R}}\right\}$ is regular in $\mathbb{R}_{+}^{\mathbb{Z}_{+}}$.

(ii) The subset $\widetilde{\mathcal{R}}_{0}:=\left\{N_{0, .}: N \in \widetilde{\mathcal{R}}\right\}$ is regular in $\mathbb{R}_{+}^{\mathbb{Z}_{+}}$.

\section{The algebra of $\mathcal{R}$-bounded generalized functions}

We adopt the standard notations and definitions of distributions and Colombeau algebra, see [11] and [10].

Let

$$
\mathcal{S}^{*}(\Omega)=\left\{f \in \mathcal{C}^{\infty}(\Omega): \forall \alpha \in \mathbb{Z}_{+}^{n}, \sup _{x \in \Omega}\left|\partial^{\alpha} f(x)\right|<\infty\right\},
$$

and $\mathcal{R}$ be a regular subset of $\mathbb{R}_{+}^{\mathbb{Z}_{+}}$, if we define

$$
\begin{aligned}
& \mathcal{E}_{\mathcal{S}^{*}}^{\mathcal{R}}(\Omega)=\left\{\left(u_{\epsilon}\right)_{\epsilon} \in \mathcal{S}^{*}(\Omega)^{I}: \exists N \in \mathcal{R}, \forall \alpha \in \mathbb{Z}_{+}^{n}, \sup _{x \in \Omega}\left|\partial^{\alpha} u_{\epsilon}(x)\right|=O\left(\epsilon^{-N_{|\alpha|}}\right), \epsilon \rightarrow 0\right\}, \\
& \mathcal{N}_{\mathcal{S}^{*}}^{\mathcal{R}}(\Omega)=\left\{\left(u_{\epsilon}\right)_{\epsilon} \in \mathcal{S}^{*}(\Omega)^{I}: \forall N \in \mathcal{R}, \forall \alpha \in \mathbb{Z}_{+}^{n}, \sup _{x \in \Omega}\left|\partial^{\alpha} u_{\epsilon}(x)\right|=O\left(\epsilon^{N_{|\alpha|}}\right), \epsilon \rightarrow 0\right\},
\end{aligned}
$$

where $I=] 0,1]$, then the properties of $\mathcal{E}_{\mathcal{S}^{*}}^{\mathcal{R}}(\Omega)$ and $\mathcal{N}_{\mathcal{S}^{*}}^{\mathcal{R}}(\Omega)$ are given by the following results.

Proposition 3.1. (i) The space $\mathcal{E}_{\mathcal{S}^{*}}^{\mathcal{R}}(\Omega)$ is a subalgebra of $\mathcal{S}^{*}(\Omega)^{I}$.

(ii) The space $\mathcal{N}_{\mathcal{S}^{*}}^{\mathcal{R}}(\Omega)$ is an ideal of $\mathcal{E}_{\mathcal{S}^{*}}^{\mathcal{R}}(\Omega)$.

(iii) We have $\mathcal{N}_{\mathcal{S}^{*}}^{\mathcal{R}}(\Omega)=\mathcal{N}_{\mathcal{S}^{*}}(\Omega)$, where

$\mathcal{N}_{\mathcal{S}^{*}}(\Omega)=\left\{\left(u_{\epsilon}\right)_{\epsilon} \in \mathcal{S}^{*}(\Omega)^{I}: \forall m \in \mathbb{Z}_{+}, \forall \alpha \in \mathbb{Z}_{+}^{n}, \sup _{x \in \Omega}\left|\partial^{\alpha} u_{\epsilon}(x)\right|=O\left(\epsilon^{m}\right), \epsilon \rightarrow 0\right\}$.

Proof. Follows easily from the definitions and standard arguments of Colombeau algebras, see [10].

Definition. An open subset $\Omega$ of $\mathbb{R}^{n}$ is said to be a box, if

$$
\Omega=\mathbf{I}_{1} \times \mathbf{I}_{2} \times \cdots \times \mathbf{I}_{n},
$$

where each $\mathbf{I}_{i}$ is a finite or infinite open interval of $\mathbb{R}$. 

box.

We have also the null characterization of the ideal $\mathcal{N}_{\mathcal{S}^{*}}(\Omega)$ provided $\Omega$ is a

Proposition 3.2. Let $\Omega$ be a box. Then an element $\left(u_{\epsilon}\right)_{\epsilon} \in \mathcal{E}_{\mathcal{S}^{*}}^{\mathcal{R}}(\Omega)$ belongs to $\mathcal{N}_{\mathcal{S}^{*}}(\Omega)$ if and only if the following condition is satisfied,

$$
\forall m \in \mathbb{Z}_{+}, \sup _{x \in \Omega}\left|u_{\epsilon}(x)\right|=O\left(\epsilon^{m}\right), \epsilon \rightarrow 0 .
$$

Proof. Suppose that $\left(u_{\epsilon}\right)_{\epsilon} \in \mathcal{E}_{\mathcal{S}^{*}}^{\mathcal{R}}(\Omega)$ satisfies $(2)$. It suffices to show that $\left(\partial_{i} u_{\epsilon}\right)_{\epsilon}$ satisfies the $\mathcal{N}_{\mathcal{S}^{*}}(\Omega)$ estimates for all $i=1, \ldots, n$. Suppose that $u_{\epsilon}$ is real valued, in the complex case, we shall carry out the following calculus separately on its real and imaginary part. Let $m \in \mathbb{Z}_{+}$, we have to show that

$$
\sup _{x \in \Omega}\left|\partial_{i} u_{\epsilon}(x)\right|=O\left(\epsilon^{m}\right), \epsilon \rightarrow 0 .
$$

Since $\left(u_{\epsilon}\right)_{\epsilon} \in \mathcal{E}_{\mathcal{S}^{*}}^{\mathcal{R}}(\Omega)$, then

$$
\exists N \in \mathcal{R}, \sup _{x \in \Omega}\left|\partial_{i}^{2} u_{\epsilon}(x)\right|=O\left(\epsilon^{-N_{2}}\right), \epsilon \rightarrow 0 .
$$

Since $\left(u_{\epsilon}\right)_{\epsilon}$ satisfies $(2)$, we have

$$
\sup _{x \in \Omega}\left|u_{\epsilon}(x)\right|=O\left(\epsilon^{N_{2}+2 m}\right), \epsilon \rightarrow 0 .
$$

By Taylor's formula, we have

$$
u_{\epsilon}\left(x+\epsilon^{N_{2}+m} e_{i}\right)=u_{\epsilon}(x)+\partial_{i} u_{\epsilon}(x) \epsilon^{N_{2}+m}+\frac{1}{2} \partial_{i}^{2} u_{\epsilon}\left(x+\theta \epsilon^{N_{2}+m} e_{i}\right) \epsilon^{2\left(N_{2}+m\right)} \text {, }
$$

where $\theta \in] 0,1[$ and $\epsilon$ is sufficiently small, as $\Omega$ is a box. It follows that

$$
\begin{aligned}
\left|\partial_{i} u_{\epsilon}(x)\right| \leq & \underbrace{\left|u_{\epsilon}\left(x+\epsilon^{N_{2}+m} e_{i}\right)\right| \epsilon^{-N_{2}-m}}_{(*)}+\underbrace{\left|u_{\epsilon}(x)\right| \epsilon^{-N_{2}-m}}_{(* *)}+ \\
& +\underbrace{\epsilon^{N_{2}+m}\left|\partial_{i}^{2} u_{\epsilon}\left(x+\theta \epsilon^{N_{2}+m} e_{i}\right)\right|}_{(* *)} .
\end{aligned}
$$

From (4), we have that $(*)$ and $(* *)$ are of order $O\left(\epsilon^{m}\right), \epsilon \rightarrow 0$, and from (3), we have that $(* * *)$ is of order $O\left(\epsilon^{m}\right), \epsilon \rightarrow 0$.

Definition. Let $\mathcal{R}$ be a regular subset of $\mathbb{R}_{+}^{\mathbb{Z}_{+}}$, the algebra of $\mathcal{R}$-bounded generalized functions, denoted by $\mathcal{G}_{\mathcal{S}^{*}}^{\mathcal{R}}(\Omega)$, is the quotient algebra

$$
\mathcal{G}_{\mathcal{S}^{*}}^{\mathcal{R}}(\Omega)=\frac{\mathcal{E}_{\mathcal{S}^{*}}^{\mathcal{R}}(\Omega)}{\mathcal{N}_{\mathcal{S}^{*}}(\Omega)} .
$$

Remark 3.3. When $\mathcal{R}$ is the set of all positive sequences, the algebra $\mathcal{G}_{\mathcal{S}^{*}}^{\mathcal{R}}(\Omega)$ is denoted by $\mathcal{G}_{L^{\infty}}(\Omega)$ in [3], this algebra is constructed on the differential algebra $D_{L^{\infty}}(\Omega)$ of Schwartz [16]. So it is more correct to write $\mathcal{G}_{L^{\infty}}^{\mathcal{R}}(\Omega)$ instead of $\mathcal{G}_{\mathcal{S}^{*}}^{\mathcal{R}}(\Omega)$. 


\section{The algebra of $\mathcal{R}$-roughly decreasing generalized functions}

Let

$$
\mathcal{S}_{*}(\Omega)=\left\{f \in \mathcal{C}^{\infty}(\Omega): \forall \beta \in \mathbb{Z}_{+}^{n}, \sup _{x \in \Omega}\left|x^{\beta} f(x)\right|<\infty\right\},
$$

and $\mathcal{R}$ be a regular subset of $\mathbb{R}_{+}^{\mathbb{Z}_{+}}$, if we define

$$
\begin{aligned}
\mathcal{E}_{\mathcal{S}_{*}}^{\mathcal{R}}(\Omega) & =\left\{\begin{array}{c}
\left(u_{\epsilon}\right)_{\epsilon} \in \mathcal{S}_{*}(\Omega)^{I}: \exists N \in \mathcal{R}, \forall \beta \in \mathbb{Z}_{+}^{n}, \\
\sup _{x \in \Omega}\left|x^{\beta} u_{\epsilon}(x)\right|=O\left(\epsilon^{-N_{|\beta|}}\right), \epsilon \rightarrow 0
\end{array}\right\}, \\
\mathcal{N}_{\mathcal{S}_{*}}^{\mathcal{R}}(\Omega) & =\left\{\begin{array}{c}
\left(u_{\epsilon}\right)_{\epsilon} \in \mathcal{S}_{*}(\Omega)^{I}: \forall N \in \mathcal{R}, \forall \beta \in \mathbb{Z}_{+}^{n}, \\
\sup _{x \in \Omega}\left|x^{\beta} u_{\epsilon}(x)\right|=O\left(\epsilon^{N_{|\beta|}}\right), \epsilon \rightarrow 0
\end{array}\right\},
\end{aligned}
$$

then the following properties of $\mathcal{E}_{\mathcal{S}_{*}}^{\mathcal{R}}(\Omega)$ and $\mathcal{N}_{\mathcal{S}_{*}}^{\mathcal{R}}(\Omega)$ are easy to verify.

Proposition 4.1. (i) The space $\mathcal{E}_{\mathcal{S}_{*}^{\mathcal{R}}}(\Omega)$ is a subalgebra of $\mathcal{S}_{*}(\Omega)^{I}$.

(ii) The space $\mathcal{N}_{\mathcal{S}_{*}}^{\mathcal{R}}(\Omega)$ is an ideal of $\mathcal{E}_{\mathcal{S}_{*}}^{\mathcal{R}}(\Omega)$.

(iii) We have $\mathcal{N}_{\mathcal{S}_{*}}^{\mathcal{R}}(\Omega)=\mathcal{N}_{\mathcal{S}_{*}}(\Omega)$, where

$$
\mathcal{N}_{\mathcal{S}_{*}}(\Omega)=\left\{\begin{array}{c}
\left(u_{\epsilon}\right)_{\epsilon} \in \mathcal{S}_{*}(\Omega)^{I}: \forall m \in \mathbb{Z}_{+}, \forall \beta \in \mathbb{Z}_{+}^{n}, \\
\sup _{x \in \Omega}\left|x^{\beta} u_{\epsilon}(x)\right|=O\left(\epsilon^{m}\right), \epsilon \rightarrow 0
\end{array}\right\} .
$$

The following proposition characterizes $\mathcal{N}_{\mathcal{S}_{*}}(\Omega)$.

Proposition 4.2. Let $\left(u_{\epsilon}\right)_{\epsilon} \in \mathcal{E}_{\mathcal{S}_{*}^{\mathcal{R}}}(\Omega)$. Then $\left(u_{\epsilon}\right)_{\epsilon} \in \mathcal{N}_{\mathcal{S}_{*}}(\Omega)$ if and only if the following condition is satisfied,

$$
\forall m \in \mathbb{Z}_{+}, \sup _{x \in \Omega}\left|u_{\epsilon}(x)\right|=O\left(\epsilon^{m}\right), \epsilon \rightarrow 0 .
$$

Proof. Suppose that $\left(u_{\epsilon}\right)_{\epsilon} \in \mathcal{E}_{\mathcal{S}_{*}}^{\mathcal{R}}(\Omega)$ satisfies $(6)$. Since $\left(u_{\epsilon}\right)_{\epsilon} \in \mathcal{E}_{\mathcal{S}_{*}}^{\mathcal{R}}(\Omega)$, then $\exists N \in \mathcal{R}, \forall \beta \in \mathbb{Z}_{+}^{n}$,

$$
\sup _{x \in \Omega}\left|x^{2 \beta} u_{\epsilon}(x)\right|=O\left(\epsilon^{-N_{2|\beta|}}\right), \epsilon \rightarrow 0 .
$$

From (6), for all $m \in \mathbb{Z}_{+}$we have

$$
\sup _{x \in \Omega}\left|u_{\epsilon}(x)\right|=O\left(\epsilon^{2 m+N_{2|\beta|}}\right), \epsilon \rightarrow 0 .
$$

Therefore $\forall x \in \Omega$,

hence

$$
\left|x^{\beta} u_{\epsilon}(x)\right|^{2}=\left|x^{2 \beta} u_{\epsilon}(x)\right|\left|u_{\epsilon}(x)\right|=O\left(\epsilon^{2 m}\right), \epsilon \rightarrow 0,
$$

$$
\left|x^{\beta} u_{\epsilon}(x)\right|=O\left(\epsilon^{m}\right), \epsilon \rightarrow 0 .
$$

Definition. Let $\mathcal{R}$ be a regular subset of $\mathbb{R}_{+}^{\mathbb{Z}_{+}}$, the algebra of $\mathcal{R}$-roughly decreasing generalized functions, denoted by $\mathcal{G}_{\mathcal{S}_{*}}^{\mathcal{R}}(\Omega)$, is the quotient algebra

$$
\mathcal{G}_{\mathcal{S}_{*}}^{\mathcal{R}}(\Omega)=\frac{\mathcal{E}_{\mathcal{S}_{*}}^{\mathcal{R}}(\Omega)}{\mathcal{N}_{\mathcal{S}_{*}}(\Omega)} .
$$


Remark 4.3. The $C^{\infty}$ regularity in the definition of elements of $\mathcal{G}_{\mathcal{S}_{*}}^{\mathcal{R}}(\Omega)$ is not in fact needed in the proof of the principal results of this work.

\section{The algebra of $\widetilde{\mathcal{R}}$-rapidly decreasing generalized functions}

Recall

$$
\mathcal{S}(\Omega)=\left\{f \in \mathcal{C}^{\infty}(\Omega): \forall(\alpha, \beta) \in \mathbb{Z}_{+}^{2 n}, \sup _{x \in \Omega}\left|x^{\beta} \partial^{\alpha} f(x)\right|<\infty\right\},
$$

the space of rapidly decreasing functions on $\Omega$, and let $\widetilde{\mathcal{R}}$ be a regular subset of $\mathbb{R}_{+}^{\mathbb{Z}_{+}^{2}}$, if we define

$$
\begin{gathered}
\mathcal{E}_{\mathcal{S}}^{\widetilde{\mathcal{R}}}(\Omega)=\left\{\begin{array}{c}
\left(u_{\epsilon}\right)_{\epsilon} \in \mathcal{S}(\Omega)^{I}: \exists N \in \widetilde{\mathcal{R}}, \forall(\alpha, \beta) \in \mathbb{Z}_{+}^{2 n}, \\
\sup _{x \in \Omega}\left|x^{\beta} \partial^{\alpha} u_{\epsilon}(x)\right|=O\left(\epsilon^{\left.-N_{|\alpha|,|\beta|}\right), \epsilon \rightarrow 0}\right.
\end{array}\right\}, \\
\mathcal{N}_{\mathcal{S}}^{\widetilde{\mathcal{R}}}(\Omega)=\left\{\begin{array}{c}
\left(u_{\epsilon}\right)_{\epsilon} \in \mathcal{S}(\Omega)^{I}: \forall N \in \widetilde{\mathcal{R}}, \forall(\alpha, \beta) \in \mathbb{Z}_{+}^{2 n}, \\
\sup _{x \in \Omega}\left|x^{\beta} \partial^{\alpha} u_{\epsilon}(x)\right|=O\left(\epsilon^{N_{|\alpha|,|\beta|}}\right), \epsilon \rightarrow 0
\end{array}\right\},
\end{gathered}
$$

then we have the following results.

Proposition 5.1. We have the following assertions:

(i) The space $\mathcal{E}_{\mathcal{S}}^{\widetilde{\mathcal{R}}}(\Omega)$ is a subalgebra of $\mathcal{S}(\Omega)^{I}$.

(ii) The space $\mathcal{N}_{\mathcal{S}}^{\widetilde{\mathcal{R}}}(\Omega)$ is an ideal of $\mathcal{E}_{\mathcal{S}}^{\widetilde{\mathcal{R}}}(\Omega)$.

(iii) We have $\mathcal{N}_{\mathcal{S}}^{\widetilde{\mathcal{R}}}(\Omega)=\mathcal{N}_{\mathcal{S}}(\Omega)$, where

$$
\mathcal{N}_{\mathcal{S}}(\Omega)=\left\{\begin{array}{c}
\left(u_{\epsilon}\right)_{\epsilon} \in \mathcal{S}(\Omega)^{I}: \forall m \in \mathbb{Z}_{+}, \forall(\alpha, \beta) \in \mathbb{Z}_{+}^{2 n}, \\
\sup _{x \in \Omega}\left|x^{\beta} \partial^{\alpha} u_{\epsilon}(x)\right|=O\left(\epsilon^{m}\right), \epsilon \rightarrow 0
\end{array}\right\} .
$$

Proof. The proof is not difficult, it follows from the properties of the set $\widetilde{\mathcal{R}}$.

Definition. Let $\widetilde{\mathcal{R}}$ be a regular subset of $\mathbb{R}_{+}^{\mathbb{Z}_{+}^{2}}$, the algebra of $\widetilde{\mathcal{R}}$-rapidly decreasing generalized functions on $\Omega$, denoted by $\mathcal{G}_{\mathcal{S}}^{\widetilde{\mathcal{R}}}(\Omega)$, is the quotient algebra

$$
\mathcal{G}_{\mathcal{S}}^{\widetilde{\mathcal{R}}}(\Omega)=\frac{\mathcal{E}_{\mathcal{S}}^{\widetilde{\mathcal{R}}}(\Omega)}{\mathcal{N}_{\mathcal{S}}(\Omega)}
$$

Example 5.2. (i) For $\widetilde{\mathcal{R}}=\mathbb{R}_{+}^{\mathbb{Z}_{+}^{2}}$, we obtain the algebra $\mathcal{G}_{\mathcal{S}}(\Omega)$ of rapidly decreasing generalized functions on $\Omega$, see $[10]$.

(ii) For $\widetilde{\mathcal{R}}=\widetilde{\mathcal{B}}$, we obtain the algebra $\mathcal{G}_{\mathcal{S}}^{\infty}(\Omega)$ of regular rapidly decreasing generalized functions on $\Omega$, see [7]. 


\section{Characterization of $\widetilde{\mathcal{R}}$-rapidly decreasing generalized functions}

Let us mention that the theorem of [4] can be extended to an open subset $\Omega$ of $\mathbb{R}^{n}$, provided $\Omega$ is a box.

Proposition 6.1. If $\Omega$ is a box of $\mathbb{R}^{n}$, then

$$
\mathcal{S}(\Omega)=\mathcal{S}^{*}(\Omega) \cap \mathcal{S}_{*}(\Omega) .
$$

Proof. The proof is the same as in [4], noting that in the Taylor's expansion, the hypothesis that $\Omega$ is a box assures that $\left(x_{1}+h, x^{\prime}\right)$ stays in $\Omega$ for all $\left(x_{1}, x^{\prime}\right) \in \Omega$ and $h>0$ sufficiently small.

The principal result of this section is an extension of (7) to the algebra of $\widetilde{\mathcal{R}}$-rapidly decreasing generalized functions. It is the first characterization of the algebra $\mathcal{G}_{\mathcal{S}}^{\widetilde{\mathcal{R}}}(\Omega)$ in the spirit of $(7)$.

Theorem 6.2. If $\Omega$ is a box, then

$$
\mathcal{G}_{\mathcal{S}}^{\widetilde{\mathcal{R}}}(\Omega)=\mathcal{G}_{\mathcal{S}_{*}}^{\widetilde{\mathcal{R}}_{0}}(\Omega) \cap \mathcal{G}_{\mathcal{S}^{*}}^{\widetilde{\mathcal{R}}^{0}}(\Omega)
$$

Proof. We have to show that $\mathcal{E}_{\mathcal{S}}^{\widetilde{\mathcal{R}}}(\Omega)=\mathcal{E}_{\mathcal{S}_{*}}^{\widetilde{\mathcal{R}}_{0}}(\Omega) \cap \mathcal{E}_{\mathcal{S}^{*}}^{\widetilde{\mathcal{R}}^{0}}(\Omega)$ and $\mathcal{N}_{\mathcal{S}}(\Omega)=$ $\mathcal{N}_{\mathcal{S}_{*}}(\Omega) \cap \mathcal{N}_{\mathcal{S}^{*}}(\Omega)$. The inclusions $\mathcal{E}_{\mathcal{S}}^{\widetilde{\mathcal{R}}}(\Omega) \subset \mathcal{E}_{\mathcal{S}_{*}} \widetilde{\mathcal{R}}_{0}(\Omega) \cap \mathcal{E}_{\mathcal{S}^{*}} \widetilde{\mathcal{R}}^{0}(\Omega)$ and $\mathcal{N}_{\mathcal{S}}(\Omega) \subset$ $\mathcal{N}_{\mathcal{S}_{*}}(\Omega) \cap \mathcal{N}_{\mathcal{S}^{*}}(\Omega)$ are obvious. In order to show the inclusion $\mathcal{E}_{\mathcal{S}_{*}}^{\widetilde{\mathcal{R}}_{0}}(\Omega) \cap$ $\mathcal{E}_{\mathcal{S}^{*}}^{\widetilde{\mathcal{R}}^{0}}(\Omega) \subset \mathcal{E}_{\mathcal{S}}^{\widetilde{\mathcal{R}}}(\Omega)$, let $\left(u_{\epsilon}\right)_{\epsilon} \in \mathcal{E}_{\mathcal{S}_{*}}^{\widetilde{\mathcal{R}}_{0}}(\Omega) \cap \mathcal{E}_{\mathcal{S}^{*}}^{\widetilde{\mathcal{R}}^{0}}(\Omega)$, then $\left(u_{\epsilon}\right)_{\epsilon} \in \mathcal{S}^{*}(\Omega)^{I} \cap$ $\mathcal{S}_{*}(\Omega)^{I}=\mathcal{S}(\Omega)^{I}$. In order to show that $\left(u_{\epsilon}\right)_{\epsilon}$ satisfies the estimates of $\mathcal{E}_{\mathcal{S}}^{\widetilde{\mathcal{R}}}(\Omega)$, set $x=\left(x_{1}, x^{\prime}\right) \in \mathbf{I}_{1} \times\left(\mathbf{I}_{2} \times \mathbf{I}_{3} \times \cdots \times \mathbf{I}_{n}\right):=\Omega$ and consider in first the case $x_{1}>0$. For $h>0$ sufficiently small, the Taylor's expansion of $u_{\epsilon}$ with respect to $x_{1}$ gives

$$
u_{\epsilon}\left(x_{1}+h, x^{\prime}\right)=u_{\epsilon}\left(x_{1}, x^{\prime}\right)+h \partial_{1} u_{\epsilon}\left(x_{1}, x^{\prime}\right)+\frac{h^{2}}{2} \partial_{1}^{2} u_{\epsilon}\left(\xi, x^{\prime}\right)
$$

for $\xi \in] x_{1}, x_{1}+h\left[\right.$. The hypothesis $\left(u_{\epsilon}\right)_{\epsilon} \in \mathcal{E}_{\mathcal{S}_{*}}^{\widetilde{\mathcal{R}}_{0}}(\Omega) \cap \mathcal{E}_{\mathcal{S}^{*}}^{\widetilde{\mathcal{R}}^{0}}(\Omega)$ gives

$$
\begin{gathered}
\exists L \in \widetilde{\mathcal{R}}_{0} ; \forall k \in \mathbb{Z}_{+}, \sup _{x_{1}>0}\left(1+|x|^{2}\right)^{k}\left|u_{\epsilon}(x)\right|=O\left(\epsilon^{-L_{k}}\right), \epsilon \rightarrow 0, \\
\sup _{x_{1}>0}\left(1+|x|^{2}\right)^{k}\left|u_{\epsilon}\left(x_{1}+h, x^{\prime}\right)\right| \leq \sup _{x_{1}>0}\left(1+\left|\left(x_{1}+h, x^{\prime}\right)\right|^{2}\right)^{k}\left|u_{\epsilon}\left(x_{1}+h, x^{\prime}\right)\right| \\
=O\left(\epsilon^{-L_{k}}\right), \epsilon \rightarrow 0, \\
\exists M \in \widetilde{\mathcal{R}}^{0}, \sup _{x_{1}>0}\left|\partial_{1}^{2} u_{\epsilon}(x)\right|=O\left(\epsilon^{-M_{2}}\right), \epsilon \rightarrow 0 .
\end{gathered}
$$

It follows from (9) that

$$
\left|\partial_{1} u_{\epsilon}\left(x_{1}, x^{\prime}\right)\right| \leq \frac{1}{h}\left[\left|u_{\epsilon}\left(x_{1}+h, x^{\prime}\right)\right|+\left|u_{\epsilon}\left(x_{1}, x^{\prime}\right)\right|\right]+\frac{h}{2}\left|\partial_{1}^{2} u_{\epsilon}\left(\xi, x^{\prime}\right)\right| .
$$


Therefore

$$
\sup _{x_{1}>0}\left(1+|x|^{2}\right)^{k}\left|\partial_{1} u_{\epsilon}(x)\right|^{2}=O\left(\epsilon^{-L_{k}-M_{2}}\right), \epsilon \rightarrow 0 .
$$

From $(\widetilde{R} 3)$ of Definition, there exists $N^{\prime} \in \widetilde{\mathcal{R}}$ such that

$$
L_{k}+M_{2} \leq N_{2, k}^{\prime} \text {. }
$$

Consequently

$$
\sup _{x_{1}>0}\left(1+|x|^{2}\right)^{k}\left|\partial_{1} u_{\epsilon}(x)\right|^{2}=O\left(\epsilon^{-N_{2, k}^{\prime}}\right), \epsilon \rightarrow 0 .
$$

So if $\beta \in \mathbb{Z}_{+}^{n}$, then

$$
\sup _{x_{1}>0}\left|x^{\beta} \partial_{1} u_{\epsilon}(x)\right|^{2} \leq C \sup _{x_{1}>0}\left(1+|x|^{2}\right)^{|\beta|}\left|\partial_{1} u_{\epsilon}(x)\right|^{2}=O\left(\epsilon^{-N_{2,|\beta|}^{\prime}}\right), \epsilon \rightarrow 0 .
$$

If $x_{1}<0$, one considers $v_{\epsilon}$ such that $v_{\epsilon}(x)=u_{\epsilon}\left(-x_{1}, x^{\prime}\right)$. We see that $\left(v_{\epsilon}\right)_{\epsilon} \in$ $\mathcal{E}_{\mathcal{S}_{*}}^{\widetilde{\mathcal{R}}_{0}}(\Omega) \cap \mathcal{E}_{\mathcal{S}^{*}}^{\widetilde{\mathcal{R}}^{0}}(\Omega)$ and consequently the precedent argument gives the existence of $N " \in \widetilde{\mathcal{R}}$ such that

$$
\sup _{x_{1}>0}\left|x^{\beta} \partial_{1} v_{\epsilon}(x)\right|^{2}=\sup _{x_{1}<0}\left|x^{\beta} \partial_{1} u_{\epsilon}(x)\right|^{2}=O\left(\epsilon^{\left.-N_{2,|\beta|}\right)}\right), \epsilon \rightarrow 0 .
$$

Now from $(\widetilde{R} 1)$ and $(\widetilde{R} 2)$ of Definition, there exists $N \in \widetilde{\mathcal{R}}$ such that

$$
\max \left(N_{2,|\beta|}^{\prime}, N^{\prime \prime}{ }_{2,|\beta|}\right) \leq N_{1,|\beta|},
$$

consequently

$$
\sup _{x \in \Omega}\left|x^{\beta} \partial_{1} u_{\epsilon}(x)\right|=O\left(\epsilon^{-N_{1,|\beta|}}\right), \epsilon \rightarrow 0 .
$$

In a similar way, we show

$$
\exists N \in \widetilde{\mathcal{R}} ; \forall \beta \in \mathbb{Z}_{+}^{n}, \sup _{x \in \Omega}\left|x^{\beta} \partial_{i} u_{\epsilon}(x)\right|=O\left(\epsilon^{-N_{1,|\beta|}}\right), i=2, \ldots, n .
$$

Therefore, by induction, we obtain

$$
\exists N \in \widetilde{\mathcal{R}} ; \forall \alpha \in \mathbb{Z}_{+}^{n}, \forall \beta \in \mathbb{Z}_{+}^{n}, \sup _{x \in \Omega}\left|x^{\beta} \partial^{\alpha} u_{\epsilon}(x)\right|=O\left(\epsilon^{-N_{|\alpha|}|\beta|}\right), \epsilon \rightarrow 0,
$$

i.e., $\left(u_{\epsilon}\right)_{\epsilon} \in \mathcal{E}_{\mathcal{S}}^{\widetilde{\mathcal{R}}}(\Omega)$.

Suppose now that $\left(u_{\epsilon}\right)_{\epsilon} \in \mathcal{N}_{\mathcal{S}_{*}}(\Omega) \cap \mathcal{N}_{\mathcal{S}^{*}}(\Omega)$. Then

$$
\begin{gathered}
\forall m \in \mathbb{Z}_{+}, \forall k \in \mathbb{Z}_{+}, \sup _{x_{1}>0}\left(1+|x|^{2}\right)^{k}\left|u_{\epsilon}(x)\right|=O\left(\epsilon^{\frac{m}{2}}\right), \epsilon \rightarrow 0, \\
\sup _{x_{1}>0}\left(1+|x|^{2}\right)^{k}\left|u_{\epsilon}\left(x_{1}+h, x^{\prime}\right)\right| \leq \sup _{x_{1}>0}\left(1+\left|\left(x_{1}+h, x^{\prime}\right)\right|^{2}\right)^{k}\left|u_{\epsilon}\left(x_{1}+h, x^{\prime}\right)\right| \\
=O\left(\epsilon^{\frac{m}{2}}\right), \epsilon \rightarrow 0, \\
\forall m \in \mathbb{Z}_{+} ; \sup _{x_{1}>0}\left|\partial_{1}^{2} u_{\epsilon}(x)\right|=O\left(\epsilon^{\frac{m}{2}}\right), \epsilon \rightarrow 0 .
\end{gathered}
$$


It follows from (9) that

$$
\sup _{x_{1}>0}\left(1+|x|^{2}\right)^{k}\left|\partial_{1} u_{\epsilon}(x)\right|^{2}=O\left(\epsilon^{m}\right), \epsilon \rightarrow 0 .
$$

Consequently, if $\beta \in \mathbb{Z}_{+}^{n}$, then

$$
\sup _{x_{1}>0}\left|x^{\beta} \partial_{1} u_{\epsilon}(x)\right|^{2} \leq C_{1} \sup _{x_{1}>0}\left(1+|x|^{2}\right)^{|\beta|}\left|\partial_{1} u_{\epsilon}(x)\right|^{2}=O\left(\epsilon^{m}\right), \epsilon \rightarrow 0 .
$$

If $x_{1}<0$, one considers $v_{\epsilon}$ such that $v_{\epsilon}(x)=u_{\epsilon}\left(-x_{1}, x^{\prime}\right)$ as above, then we obtain

$$
\sup _{x_{1}>0}\left|x^{\beta} \partial_{1} v_{\epsilon}(x)\right|^{2}=\sup _{x_{1}<0}\left|x^{\beta} \partial_{1} u_{\epsilon}(x)\right|^{2}=O\left(\epsilon^{m}\right), \epsilon \rightarrow 0 .
$$

Therefore, by induction, we have

$$
\forall m \in \mathbb{Z}_{+}, \forall \alpha \in \mathbb{Z}_{+}^{n}, \forall \beta \in \mathbb{Z}_{+}^{n}, \sup _{x \in \Omega}\left|x^{\beta} \partial^{\alpha} u_{\epsilon}(x)\right|=O\left(\epsilon^{m}\right), \epsilon \rightarrow 0 .
$$

Thus $\mathcal{N}_{\mathcal{S}_{*}}(\Omega) \cap \mathcal{N}_{\mathcal{S}^{*}}(\Omega) \subset \mathcal{N}_{\mathcal{S}}(\Omega)$ and consequently $\mathcal{G}_{\mathcal{S}}^{\widetilde{\mathcal{R}}}(\Omega)=\mathcal{G}_{\mathcal{S}_{*}}^{\widetilde{\mathcal{R}}_{0}}(\Omega) \cap$ $\mathcal{G}_{\mathcal{S}^{*}}^{\widetilde{\mathcal{R}}^{0}}(\Omega)$.

Propositions 3.2 and 4.2 give the following result characterizing the negligible elements of the algebra $\mathcal{G}_{\mathcal{S}}^{\widetilde{\mathcal{R}}}(\Omega)$.

Corollary 6.3. Let $\Omega$ be a box. Then an element $\left(u_{\epsilon}\right)_{\epsilon} \in \mathcal{E}_{\mathcal{S}}^{\widetilde{\mathcal{R}}}(\Omega)$ is in $\mathcal{N}_{\mathcal{S}}(\Omega)$ if and only if the following condition is satisfied,

$$
\forall m \in \mathbb{Z}_{+}, \sup _{x \in \Omega}\left|u_{\epsilon}(x)\right|=O\left(\epsilon^{m}\right), \epsilon \rightarrow 0 .
$$

Theorem 6.2 gives the following corollaries characterizing the algebra of rapidly decreasing generalized functions $\mathcal{G}_{\mathcal{S}}$ and the algebra of regular rapidly decreasing generalized functions $\mathcal{G}_{\mathcal{S}}^{\infty}$.

Corollary 6.4. (i) When $\widetilde{\mathcal{R}}=\mathbb{R}_{+}^{\mathbb{Z}_{+}^{2}}$ we obtain $\mathcal{G}_{\mathcal{S}}^{\mathbb{R}_{+}^{\mathbb{Z}_{+}^{2}}}=\mathcal{G}_{\mathcal{S}}$ and we have

$$
\mathcal{G}_{\mathcal{S}}=\mathcal{G}_{\mathcal{S}^{*}} \cap \mathcal{G}_{\mathcal{S}_{*}},
$$

where

$$
\mathcal{G}_{\mathcal{S}^{*}}:=\frac{\left\{\left(u_{\epsilon}\right)_{\epsilon} \in \mathcal{S}^{* I}: \forall \alpha \in \mathbb{Z}_{+}^{n}, \exists m \in \mathbb{Z}_{+}, \sup _{x \in \mathbb{R}^{n}}\left|\partial^{\alpha} u_{\epsilon}(x)\right|=O\left(\epsilon^{-m}\right), \epsilon \rightarrow 0\right\}}{\left\{\left(u_{\epsilon}\right)_{\epsilon} \in \mathcal{S}^{* I}: \forall \alpha \in \mathbb{Z}_{+}^{n}, \forall m \in \mathbb{Z}_{+}, \sup _{x \in \mathbb{R}^{n}}\left|\partial^{\alpha} u_{\epsilon}(x)\right|=O\left(\epsilon^{m}\right), \epsilon \rightarrow 0\right\}}
$$

and

$$
\mathcal{G}_{\mathcal{S}_{*}}:=\frac{\left\{\left(u_{\epsilon}\right)_{\epsilon} \in \mathcal{S}_{*}^{I}: \forall \beta \in \mathbb{Z}_{+}^{n}, \exists m \in \mathbb{Z}_{+}, \sup _{x \in \mathbb{R}^{n}}\left|x^{\beta} u_{\epsilon}(x)\right|=O\left(\epsilon^{-m}\right), \epsilon \rightarrow 0\right\}}{\left\{\left(u_{\epsilon}\right)_{\epsilon} \in \mathcal{S}_{*}^{I}: \forall \beta \in \mathbb{Z}_{+}^{n}, \forall m \in \mathbb{Z}_{+}, \sup _{x \in \mathbb{R}^{n}}\left|x^{\beta} u_{\epsilon}(x)\right|=O\left(\epsilon^{m}\right), \epsilon \rightarrow 0\right\}} .
$$


(ii) When $\widetilde{\mathcal{R}}=\widetilde{\mathcal{B}}$ we obtain $\mathcal{G}_{\mathcal{S}}^{\widetilde{\mathcal{B}}}=\mathcal{G}_{\mathcal{S}}^{\infty}$ and we have

$$
\mathcal{G}_{\mathcal{S}}^{\infty}=\mathcal{G}_{\mathcal{S}^{*}}^{\infty} \cap \mathcal{G}_{\mathcal{S}_{*}}^{\infty},
$$

where

$$
\mathcal{G}_{\mathcal{S}^{*}}^{\infty}:=\frac{\left\{\left(u_{\epsilon}\right)_{\epsilon} \in \mathcal{S}^{* I}: \exists m \in \mathbb{Z}_{+}, \forall \alpha \in \mathbb{Z}_{+}^{n}, \sup _{x \in \mathbb{R}^{n}}\left|\partial^{\alpha} u_{\epsilon}(x)\right|=O\left(\epsilon^{-m}\right), \epsilon \rightarrow 0\right\}}{\left\{\left(u_{\epsilon}\right)_{\epsilon} \in \mathcal{S}^{* I}: \forall m \in \mathbb{Z}_{+}, \forall \alpha \in \mathbb{Z}_{+}^{n}, \sup _{x \in \mathbb{R}^{n}}\left|\partial^{\alpha} u_{\epsilon}(x)\right|=O\left(\epsilon^{m}\right), \epsilon \rightarrow 0\right\}}
$$

and

$$
\mathcal{G}_{\mathcal{S}_{*}}^{\infty}:=\frac{\left\{\left(u_{\epsilon}\right)_{\epsilon} \in \mathcal{S}_{*}^{I}: \exists m \in \mathbb{Z}_{+}, \forall \beta \in \mathbb{Z}_{+}^{n}, \sup _{x \in \mathbb{R}^{n}}\left|x^{\beta} u_{\epsilon}(x)\right|=O\left(\epsilon^{-m}\right), \epsilon \rightarrow 0\right\}}{\left\{\left(u_{\epsilon}\right)_{\epsilon} \in \mathcal{S}_{*}^{I}: \forall m \in \mathbb{Z}_{+}, \forall \beta \in \mathbb{Z}_{+}^{n}, \sup _{x \in \mathbb{R}^{n}}\left|x^{\beta} u_{\epsilon}(x)\right|=O\left(\epsilon^{m}\right), \epsilon \rightarrow 0\right\}} .
$$

\section{Characterization of $\widetilde{\mathcal{R}}$-rapidly decreasing generalized functions} via the Fourier transform

The Fourier transform of $u \in \mathcal{S}$, denoted by $\widehat{u}$ or $\mathcal{F}(u)$, is defined by

$$
\widehat{u}(\xi)=(2 \pi)^{-\frac{n}{2}} \int e^{-i x \xi} u(x) d x .
$$

Definition. The Fourier transform of $u=\left[\left(u_{\epsilon}\right)_{\epsilon}\right] \in \mathcal{G}_{\mathcal{S}}^{\widetilde{\mathcal{R}}}$, denoted by $\mathcal{F}_{\mathcal{S}}(u)$, is defined by

$$
\mathcal{F}_{\mathcal{S}}(u)=\widehat{u}=\left[\left(\widehat{u_{\epsilon}}\right)_{\epsilon}\right] \text { in } \mathcal{G}_{\mathcal{S}}^{\widetilde{\mathcal{R}}} \text {. }
$$

Remark 7.1. The inverse Fourier transform of $u \in \mathcal{S}$, denoted $\widetilde{u}$ or $\mathcal{F}_{\mathcal{S}}^{-1}(u)$, is defined as usually.

The following proposition gives the main results of the Fourier transform $\mathcal{F}_{\mathcal{S}}$, its proof is standard.

Proposition 7.2. The map

$$
\mathcal{F}_{\mathcal{S}}: \mathcal{G}_{\mathcal{S}}^{\widetilde{\mathcal{R}}} \rightarrow \mathcal{G}_{\mathcal{S}}^{\widetilde{\mathcal{R}}}
$$

is an algebraic isomorphism.

Let

$$
\widehat{\mathcal{S}^{*}}=\left\{f \in \mathcal{C}^{\infty}: \forall \beta \in \mathbb{Z}_{+}^{n}, \sup _{\xi \in \mathbb{R}^{n}}\left|\xi^{\beta} \widehat{f}(\xi)\right|<\infty\right\},
$$

and let $\widetilde{\mathcal{R}}$ be a regular subset of $\mathbb{R}_{+}^{\mathbb{Z}_{+}^{2}}$, if we define

$$
\mathcal{E}_{\widetilde{\mathcal{S}}^{*}}^{\widetilde{\mathcal{R}}^{0}}=\left\{\left(u_{\epsilon}\right)_{\epsilon} \in \widehat{\mathcal{S}}^{*}: \exists N \in \widetilde{\mathcal{R}}^{0}, \forall \beta \in \mathbb{Z}_{+}^{n}, \sup _{\xi \in \mathbb{R}^{n}}\left|\xi^{\beta} \widehat{u_{\epsilon}}(\xi)\right|=O\left(\epsilon^{-N_{|\beta|}}\right), \epsilon \rightarrow 0\right\},
$$


CHARACTERIZATIONS OF RAPIDLY DECREASING GENERALIZED FUNCTIONS 401

$\mathcal{N}_{\widetilde{\mathcal{S}}^{*}}^{\widetilde{\mathcal{R}}^{0}}=\left\{\left(u_{\epsilon}\right)_{\epsilon} \in \widehat{\mathcal{S}}^{I}: \forall N \in \widetilde{\mathcal{R}}^{0}, \forall \beta \in \mathbb{Z}_{+}^{n}, \sup _{\xi \in \mathbb{R}^{n}}\left|\xi^{\beta} \widehat{u_{\epsilon}}(\xi)\right|=O\left(\epsilon^{N_{|\beta|}}\right), \epsilon \rightarrow 0\right\}$,

then the following proposition is easy to prove.

Proposition 7.3. (i) The space $\mathcal{E}^{\widetilde{\mathcal{R}}^{0}}$ is a subalgebra of $\widehat{\mathcal{S}}^{*}$.

(ii) The space $\mathcal{N}_{\widetilde{\mathcal{S}}^{*}}^{\widetilde{\mathcal{R}}^{0}}$ is an ideal of $\mathcal{E}_{\widetilde{\mathcal{S}}^{*}}^{\widetilde{\mathcal{R}}^{0}}$.

(iii) The ideal $\mathcal{N}_{\widetilde{\mathcal{S}}^{*}}=\mathcal{N}_{\widehat{\mathcal{S}^{*}}}$, where

$\mathcal{N}_{\widehat{\mathcal{S}^{*}}}:=\left\{\left(u_{\epsilon}\right)_{\epsilon} \in \widehat{\mathcal{S}}^{I}: \forall m \in \mathbb{Z}, \forall \beta \in \mathbb{Z}_{+}^{n}, \sup _{\xi \in \mathbb{R}^{n}}\left|\xi^{\beta} \widehat{u_{\epsilon}}(\xi)\right|=O\left(\epsilon^{m}\right), \epsilon \rightarrow 0\right\}$.

The following proposition characterizes $\mathcal{N}_{\widehat{\mathcal{S}^{*}}}$.

Proposition 7.4. Let $\left(u_{\epsilon}\right)_{\epsilon} \in \mathcal{E}_{\widetilde{\mathcal{S}}^{*}}^{\widetilde{\mathcal{R}}^{0}}$. Then $\left(u_{\epsilon}\right)_{\epsilon} \in \mathcal{N}_{\widetilde{\mathcal{S}}^{*}}^{\widetilde{\mathcal{R}}^{0}}$ if and only if the following condition is satisfied,

$$
\forall m \in \mathbb{Z}_{+}, \sup _{\xi \in \mathbb{R}^{n}}\left|\widehat{u_{\epsilon}}(\xi)\right|=O\left(\epsilon^{m}\right), \epsilon \rightarrow 0 .
$$

Proof. The proof is similar to that of Proposition 4.2.

Definition. The algebra $\frac{\widetilde{\mathcal{R}}^{0}}{\mathcal{S}^{*}}$ is defined as the quotient algebra

$$
\mathcal{G}_{\widetilde{\mathcal{S}^{*}}}^{\widetilde{\mathcal{R}}^{0}}=\frac{\mathcal{E}_{\widetilde{\mathcal{S}^{*}}}^{\widetilde{\mathcal{R}}^{0}}}{\mathcal{N}_{\widehat{\mathcal{S}^{*}}}} .
$$

The next theorem is the second characterization of $\mathcal{G}_{\mathcal{S}}^{\widetilde{\mathcal{R}}}$.

Theorem 7.5. We have

$$
\mathcal{G}_{\mathcal{S}}^{\widetilde{\mathcal{R}}}=\mathcal{G}_{\mathcal{S}_{*}}^{\widetilde{\mathcal{R}}_{0}} \cap \mathcal{G}_{\mathcal{\mathcal { S }}^{*}}^{\widetilde{\mathcal{R}}^{0}}
$$

Proof. Let $\left(u_{\epsilon}\right)_{\epsilon} \in \mathcal{E}^{\widetilde{\mathcal{R}}^{0}}$. It follows that

$$
\begin{aligned}
\int\left|x^{\beta} \widehat{u_{\epsilon}}(x)\right| d x & \leq C \sup _{x \in \mathbb{R}^{n}}\left(1+|x|^{2}\right)^{n}\left|x^{\beta} \widehat{u_{\epsilon}}(x)\right| \\
& =O\left(\epsilon^{-N_{|\beta|+2 n}^{\prime}}\right), \epsilon \rightarrow 0 \\
& =O\left(\epsilon^{-N_{|\beta|}}\right), \epsilon \rightarrow 0
\end{aligned}
$$

for some $N \in \widetilde{\mathcal{R}}^{0}$. The continuity of the Fourier transformation $\mathcal{F}$ from the Lebesgue space of integrable functions $\mathbb{L}^{1}$ to the Lebesgue space of essentially bounded functions $\mathbb{L}^{\infty}$ gives

$$
\left\|\partial^{\beta} u_{\epsilon}\right\|_{\mathbb{L}^{\infty}}=O\left(\epsilon^{-N_{|\beta|}}\right), \epsilon \rightarrow 0
$$


which shows that $\left(u_{\epsilon}\right)_{\epsilon} \in \mathcal{E}_{\mathcal{S}^{*}}^{\widetilde{\mathcal{R}}^{0}}$ and therefore $\mathcal{E}_{\widetilde{\mathcal{S}}^{*}}^{\widetilde{\mathcal{R}}^{0}} \subset \mathcal{E}_{\mathcal{S}^{*}}^{\widetilde{\mathcal{R}}^{0}}$. Consequently $\mathcal{E}_{\mathcal{S}_{*}}^{\widetilde{\mathcal{R}}_{0}} \cap$ $\mathcal{E}_{\widetilde{\mathcal{S}}^{*}}^{\widetilde{\mathcal{R}}^{*}} \subset \mathcal{E}_{\mathcal{S}}^{\widetilde{\mathcal{R}}}$. In order to show the inverse inclusion let us mention in first, that from [4], we have

$$
\left(u_{\epsilon}\right)_{\epsilon} \in \mathcal{S}^{I} \Longleftrightarrow\left(u_{\epsilon}\right)_{\epsilon} \in \mathcal{S}_{*}^{I} \cap \widehat{\mathcal{S}}^{I},
$$

which implies in particular that $\mathcal{S} \subset \widehat{\mathcal{S}^{*}}$. On the other hand if $\left(u_{\epsilon}\right)_{\epsilon} \in \mathcal{E}_{\mathcal{S}}^{\widetilde{\mathcal{R}}}$, then

$$
\begin{aligned}
\int\left|\partial^{\beta} u_{\epsilon}(x)\right| d x & \leq C \sup _{x \in \mathbb{R}^{n}}\left(1+|x|^{2}\right)^{n}\left|\partial^{\beta} u_{\epsilon}(x)\right|, \\
& =O\left(\epsilon^{-N_{|\beta|, 2 n}^{\prime}}\right), \epsilon \rightarrow 0, \\
& =O\left(\epsilon^{-N_{|\beta|, 0}}\right), \epsilon \rightarrow 0,
\end{aligned}
$$

for some $N \in \widetilde{\mathcal{R}}$, i.e.,

$$
\int\left|\partial^{\beta} u_{\epsilon}(x)\right| d x=O\left(\epsilon^{-N_{|\beta|}}\right), \epsilon \rightarrow 0,
$$

for some $N \in \widetilde{\mathcal{R}}^{0}$. The continuity $\mathcal{F}$ from $\mathbb{L}^{1}$ to $\mathbb{L}^{\infty}$ gives

$$
\left\|\xi^{\beta} \widehat{u}_{\epsilon}\right\|_{\mathbb{L}^{\infty}}=O\left(\epsilon^{-N_{|\beta|}}\right), \epsilon \rightarrow 0,
$$

which shows that $\left(u_{\epsilon}\right)_{\epsilon} \in \mathcal{E} \frac{\widetilde{\mathcal{R}}^{0}}{\mathcal{S}^{*}}$ and consequently $\left(u_{\epsilon}\right)_{\epsilon} \in \mathcal{E}_{\mathcal{S}_{*}}^{\widetilde{\mathcal{R}}_{0}} \cap \mathcal{E}_{\widetilde{\mathcal{S}}^{*}}^{\widetilde{\mathcal{R}}^{0}}$. Thus $\mathcal{E}_{\mathcal{S}}^{\widetilde{\mathcal{R}}} \subset \mathcal{E}_{\mathcal{S}_{*}}^{\widetilde{\mathcal{R}}_{0}} \cap \mathcal{E}_{\mathcal{\mathcal { S }}^{*}}^{\widetilde{\mathcal{R}}^{0}}$, so we have $\mathcal{E}_{\mathcal{S}}^{\widetilde{\mathcal{R}}}=\mathcal{E}_{\mathcal{S}_{*}}^{\widetilde{\mathcal{R}}_{0}} \cap \mathcal{E}_{\widetilde{\mathcal{S}}^{*}}^{\widetilde{\mathcal{R}}^{0}}$. A similar proof shows that $\mathcal{N}_{\mathcal{S}}=\mathcal{N}_{\mathcal{S}_{*}} \cap \mathcal{N}_{\widehat{\mathcal{S}}^{*}}$. Therefore $\mathcal{G}_{\mathcal{S}}^{\widetilde{\mathcal{R}}}=\mathcal{G}_{\mathcal{S}_{*}}^{\widetilde{\mathcal{S}}_{0}} \cap \mathcal{G}_{\widetilde{\mathcal{S}}^{*}}^{\widetilde{\mathcal{R}}^{0}}$.

The following corollary gives a second characterization of the space $\mathcal{N}_{\mathcal{S}}$.

Corollary 7.6. An element $\left(u_{\epsilon}\right)_{\epsilon} \in \mathcal{E}_{\mathcal{S}}^{\widetilde{\mathcal{R}}}$ is in $\mathcal{N}_{\mathcal{S}}$ if and only if the following condition is satisfied,

$$
\forall m \in \mathbb{Z}_{+}, \sup _{\xi \in \mathbb{R}^{n}}\left|\widehat{u_{\epsilon}}(\xi)\right|=O\left(\epsilon^{m}\right), \epsilon \rightarrow 0 .
$$

Proof. If $\left(u_{\epsilon}\right)_{\epsilon} \in \mathcal{E}_{\mathcal{S}}^{\widetilde{\mathcal{R}}}$, then $\left(u_{\epsilon}\right)_{\epsilon} \in \mathcal{E}_{\mathcal{S}_{*}}^{\widetilde{\mathcal{R}}_{0}} \cap \mathcal{E}_{\widetilde{\mathcal{S}}^{*}}^{\widetilde{\mathcal{R}}^{0}}$ by Theorem 7.5. From $\left(u_{\epsilon}\right)_{\epsilon} \in \mathcal{E}_{\widetilde{\mathcal{S}}^{*}}^{\widetilde{\mathcal{R}}^{0}}$ and (15), we have by Proposition 7.4 that $\left(u_{\epsilon}\right)_{\epsilon} \in \mathcal{N}_{\widehat{\mathcal{S}^{*}}}$. In order to show that $\left(u_{\epsilon}\right)_{\epsilon} \in \mathcal{N}_{\mathcal{S}_{*}}$, we have

$$
\begin{aligned}
\int\left|\widehat{u}_{\epsilon}(x)\right| d x & \leq C \sup _{x \in \mathbb{R}^{n}}\left(1+|x|^{2}\right)^{n}\left|\widehat{u}_{\epsilon}(x)\right|, \\
& =O\left(\epsilon^{m}\right), \epsilon \rightarrow 0,
\end{aligned}
$$

for all $m \in \mathbb{Z}_{+}$. The continuity of $\mathcal{F}$ from $\mathbb{L}^{1}$ to $\mathbb{L}^{\infty}$ gives

$$
\left\|u_{\epsilon}\right\|_{\mathbb{L}^{\infty}}=O\left(\epsilon^{m}\right), \epsilon \rightarrow 0,
$$

this implies, by Proposition 4.2 , that $\left(u_{\epsilon}\right)_{\epsilon} \in \mathcal{N}_{\mathcal{S}_{*}}$. Consequently $\left(u_{\epsilon}\right)_{\epsilon} \in \mathcal{N}_{\mathcal{S}_{*}} \cap$ $\mathcal{N}_{\widehat{\mathcal{S}^{*}}}=\mathcal{N}_{\mathcal{S}}$

We have also an other characterization of the algebra $\mathcal{G}_{\mathcal{S}}$. 
Corollary 7.7. We have

$$
\mathcal{G}_{\mathcal{S}}=\mathcal{G}_{\mathcal{S}_{*}} \cap \mathcal{G}_{\widehat{\mathcal{S}^{*}}}
$$

where

$\mathcal{G}_{\widehat{\mathcal{S}^{*}}}:=\frac{\left\{\left(u_{\epsilon}\right)_{\epsilon} \in \widehat{\mathcal{S}}^{I}: \forall \beta \in \mathbb{Z}_{+}^{n}, \exists m \in \mathbb{Z}_{+}, \sup _{\xi \in \mathbb{R}^{n}}\left|\xi^{\beta} \widehat{u_{\epsilon}}(\xi)\right|=O\left(\epsilon^{-m}\right), \epsilon \rightarrow 0\right\}}{\left\{\left(u_{\epsilon}\right)_{\epsilon} \in \widehat{\mathcal{S}}^{I}: \forall \beta \in \mathbb{Z}_{+}^{n}, \forall m \in \mathbb{Z}_{+}, \sup _{\xi \in \mathbb{R}^{n}}\left|\xi^{\beta} \widehat{u_{\epsilon}}(\xi)\right|=O\left(\epsilon^{m}\right), \epsilon \rightarrow 0\right\}}$.

The following result is obtained as a corollary of Theorem 7.5.

Corollary 7.8. We have

$$
\mathcal{G}_{\mathcal{S}}^{\infty}=\mathcal{G}_{\mathcal{S}_{*}}^{\infty} \cap \mathcal{G}_{\mathcal{\mathcal { S }}^{*}}^{\infty}
$$

where

$$
\mathcal{G}_{\widehat{\mathcal{S}^{*}}}^{\infty}:=\frac{\left\{\left(u_{\epsilon}\right)_{\epsilon} \in \widehat{\mathcal{S}}^{I}: \exists m \in \mathbb{Z}_{+}, \forall \beta \in \mathbb{Z}_{+}^{n}, \sup _{\xi \in \mathbb{R}^{n}}\left|\xi^{\beta} \widehat{u}_{\epsilon}(\xi)\right|=O\left(\epsilon^{-m}\right), \epsilon \rightarrow 0\right\}}{\left\{\left(u_{\epsilon}\right)_{\epsilon} \in \widehat{\mathcal{S}}^{*}: \forall m \in \mathbb{Z}_{+}, \forall \beta \in \mathbb{Z}_{+}^{n} \sup _{\xi \in \mathbb{R}^{n}}\left|\xi^{\beta} \widehat{u_{\epsilon}}(\xi)\right|=O\left(\epsilon^{m}\right), \epsilon \rightarrow 0\right\}} .
$$

\section{References}

[1] J. Alvarez and H. Obiedat, Characterizations of the Schwartz space $S$ and the BeurlingBjorck space $S_{w}$, Cubo 6 (2004), no. 4, 167-183.

[2] K. Benmeriem and C. Bouzar, Ultraregular generalized functions of Colombeau type, J. Math. Sci. Univ. Tokyo 15 (2008), no. 4, 427-447.

[3] H. A. Biagioni and M. Oberguggenberger, Generalized solutions to the Korteweg-de Vries and the regularized long-wave equations, SIAM J. Math. Anal. 23 (1992), no. 4, 923-940.

[4] J. Chung, S. Y. Chung, and D. Kim, Une caractérisation de l'espace $\mathcal{S}$ de Schwartz, C. R. Acad. Sci. Paris Sér. I Math. 316 (1993), no. 1, 23-25.

[5] J. F. Colombeau, New Generalized Functions and Multiplication of Distributions, NorthHolland Publishing Co., Amsterdam, 1984.

[6] A. Delcroix, Regular rapidly decreasing nonlinear generalized functions. Application to microlocal regularity, J. Math. Anal. Appl. 327 (2007), no. 1, 564-584.

[7] C. Garetto, Pseudo-differential operators in algebras of generalized functions and global hypoellipticity, Acta Appl. Math. 80 (2004), no. 2, 123-174.

[8] I. M. Gel'fand and G. E. Shilov, Generalized Functions, Vol. 2, Academic Press, 1967.

[9] K. Gröchenig and G. Zimmermann, Spaces of test functions via the STFT, J. Funct. Spaces Appl. 2 (2004), no. 1, 25-53.

[10] M. Grosser, M. Kunzinger, M. Oberguggenberger, and R. Steinbauer, Geometric Theory of Generalized Functions with Applications to General Relativity, Kluwer Academic Publishers, Dordrecht, 2001.

[11] L. Hörmander, Distributions Theory And Fourier Analysis, Springer, 1983.

[12] A. I. Kashpirovski, Equality of the spaces $S_{\beta}^{\alpha}$ and $S^{\alpha} \cap S_{\beta}$, Funct. Anal. Appl. 14 (1980), p. 129.

[13] M. Oberguggenberger, Regularity theory in Colombeau algebras, Bull. Cl. Sci. Math. Nat. Sci. Math. No. 31 (2006), 147-162. 
[14] N. Ortner and P. Wagner, Applications of weighted $\mathcal{D}_{L^{p}}^{\prime}$-spaces to the convolution of distributions, Bull. Polish Acad. Sci. Math. 37 (1989), no. 7-12, 579-595.

[15] Ya. V. Radyno, N. F. Tkhan, and S. Ramdan, The Fourier transformation in an algebra of new generalized functions, Dokl. Akad. Nauk 327 (1992), no. 1, 20-24; translation in Russian Acad. Sci. Dokl. Math. 46 (1993), no. 3, 414-417.

[16] L. Schwartz, Théorie des distributions, Hermann, Paris, 1966.

[17] L. Volevich and S. Gindikin, The Cauchy problem and related problems for convolution equations, Uspehi Mat. Nauk 27 (1972), no. 4(166), 65-143.

CHIKH Bouzar

OrAN-ESSENIA UNIVERSITY

ORAN 31000, ALGERIA

E-mail address: bouzar@yahoo.com; bouzar@univ-oran.dz

TAYEB SAIDI

UNIVERSITY OF BECHAR

BECHAR 08000, Algeria

E-mail address: saidi_tb@yahoo.fr 